\title{
An explicit Lagrangian finite element method for free-surface weakly compressible flows
}

\author{
Massimiliano Cremonesi · Simone Meduri • Umberto Perego • Attilio \\ Frangi
}

\begin{abstract}
In the present work, an explicit finite element approach to the solution of the Lagrangian formulation of the Navier-Stokes equations for weakly compressible fluids or fluid-like materials is investigated. The introduction of a small amount of compressibility is shown to allow for the formulation of a fast and robust explicit solver based on a Particle Finite Element Method. Newtonian and Non-Newtonian Bingham laws are considered. A barotropic equation of state completes the model relating pressure and density fields. The approach has been validated through comparison with experimental tests and numerical simulations of free surface fluid problems involving water and watersoil mixtures.
\end{abstract}

Keywords Particle Finite Element Method · volume conservation $\cdot$ alpha shape $\cdot$ remeshing

\section{Introduction}

Weakly compressible flows are frequent in nature as a small amount of compressibility exists in all the cases idealized as incompressible. In many practical circumstances, weakly compressible solvers can be conveniently used in problems that would be classified as of incompressible flow.

The physical difference between incompressible and compressible fluids is in the propagation of dilatational waves. In a quasi-incompressible medium, the speed

M. Cremonesi · S. Meduri - U. Perego · A. Frangi

Department of Civil and Environmental Engineering, Politecnico di Milano, Piazza Leonardo da Vinci 32, 20133 Milano, Italy

Tel.: +3902 23996230

Fax: +3902 23994300

E-mail: massimiliano.cremonesi@polimi.it of dilatational waves tends to infinity, while in compressible bodies the wave speed has a finite value. This is reflected also in the nature of the system of equations: hyperbolic-parabolic for the compressible case and elliptic-parabolic for the incompressible one [26].

Compressibility effects can be characterized by the Mach number defined as the ratio between the speed of the fluid and the speed of sound in the same fluid. The incompressible limit is obtained for a Mach number equal to zero. For Mach numbers much smaller than one, the weakly compressible equations asymptotically approach the incompressible Navier-Stokes equations. Consequently, for suitable small Mach numbers, the weakly compressible model well approximates the incompressible limit. This justifies the fact that a compressible solver is used to solve boundary value problems with quasi-incompressible fluids [1].

The real benefit of compressible solvers versus incompressible ones is the possibility to avoid the use of a Poisson solver. In fact, in the incompressible formulation, the continuity equation represents a constraint on velocity and combined with momentum conservation equation leads to a Poisson-like equation for pressure. Moreover, in the compressible framework, with a suitable equation of state for the pressure, it is possible to use an explicit time integration that has proved to be robust and very fast for complex hydrodynamic problems [36,13]. Furthermore, in the compressible regime, the density is adjusted to strongly respect the mass conservation equation and the typical mass variations due to the stabilization of incompressible solvers are avoided.

In the compressible framework, an equation of state is necessary to link pressure and density. Following [5, 15], in this work a modified Tait's equation is used. When a fluid is modeled with a Tait's equation, the en- 
ergy equation becomes decoupled from mass and momentum conservations [15], and, considering an isothermal flow, can be neglected.

Depending on the framework in which the balance equations for the compressible fluid are written, numerical methods can be classified in three categories: Eulerian, Lagrangian and Arbitrary Lagrangian Eulerian (ALE). In the Eulerian approach, the problem is solved on a fixed mesh through which the fluid moves; on the contrary Lagrangian methods are based on meshes that move with the fluid velocity. In the ALE approach, the velocity of the mesh is computed independently of the fluid velocity. The three approaches have been extensively used in the solution of compressible and weakly compressible problems. For example, $[6,4]$ propose an Eulerian finite volume for multi-material fluid flows, while in $[36,12]$ a Lagrangian finite element method is used to solve compressible flow problems. A discussion of the advantages and disadvantages of the Lagrangian and Eulerian approaches can be found in [3]. ALE methods try to combine the advantages of Eulerian and Lagrangian approaches (see for example $[2,17$, $40])$. In this work, we concentrate on Lagrangian approaches because they are particularly suited for free surface fluid flows.

With Smoothed-Particle Hydrodynamics (SPH) methods, weakly compressible formulations have often been proposed to model the incompressible limit. [8] presents a comparative study of the incompressible and weakly compressible solvers for SPH applications to free surface flows. Other SPH applications can be found, for example, in $[38,41]$.

Finite elements have been extensively used in the simulation of Lagrangian compressible flows. In [19] a review of stabilized finite element methods for compressible flow applications is presented. [36,37] propose a multiscale framework for the simulation of shocks hydrodynamics based on finite elements. $[12,13]$ introduce a high-order curvilinear finite element approach to solve the Euler equations in a Lagrangian moving frame. [25, 24] propose a Lagrangian finite element approach based on the Particle Finite Element Method (PFEM) to simulate underwater implosion of large air bubbles. In these works, an adaptive mesh generation based on the constrained Delaunay triangulation has been used to capture large distortions of the interface that appear in multi-fluid flows. However, all these works are limited to confined flows without free-surface, whereas in the present approach we exploit the potential of the Lagrangian framework, to solve problems in the presence of evolving free-surfaces.

As expected when using a pure Lagrangian approach, the mesh can deteriorate in time. In this work, in the spirit of the PFEM, when the mesh becomes too distorted, a Delaunay triangulation is applied to redefine element connectivities. Moreover an alpha-shape technique is used to recover the free-surface configuration $[21,34,10]$.

The PFEM has also been used in [33] for the discretization of quasi-incompressible flows. However, in that work the effect of compressibility is considered in the continuity equations, while the density is constant in all the domain and no equation of state for the pressure is necessary. In contrast, in the present approach, the density can vary with time and position and it is related to the pressure through an equation of state.

In this work, an explicit Lagrangian finite element method based on the PFEM is used to solve weakly compressible fluid problems with evolving free surfaces. Both Newtonian and non-Newtonian fluids are considered. The paper is structured as follows. In section 2 the balance equations, the constitutive laws and the equation of state are introduced. Section 3 and section 4 describe space and time discretizations respectively, while section 5 briefly summarizes the key features of the PFEM solution scheme. Finally, conclusions and future developments of the present work are highlighted in section 7 .

\section{Balance equations}

Let $\Omega_{t}$ be a domain evolving in the time interval $[0, T]$. In the initial configuration $\Omega_{0}$ let $\mathbf{X}$ be the particles position. Particles position $\mathbf{x}$ at time $t$ can be expressed as:

$\mathbf{x}=\mathbf{x}(\mathbf{X}, t)$

Defining now the velocity field $\mathbf{u}=\mathbf{u}(\mathbf{x}, t)$, the density field $\rho=\rho(\mathbf{x}, t)$ and the Cauchy stress tensor $\boldsymbol{\sigma}=$ $\boldsymbol{\sigma}(\mathbf{x}, t)$, the equations of motion for a compressible fluid can be expressed as:

$$
\begin{array}{ll}
\frac{d \rho}{d t}+\rho\left(\nabla_{\mathbf{x}} \cdot \mathbf{u}\right)=0 & \text { in } \Omega_{t} \times[0, T] \\
\rho \frac{d \mathbf{u}}{d t}=\nabla_{\mathbf{x}} \cdot \boldsymbol{\sigma}+\rho \mathbf{b} & \text { in } \Omega_{t} \times[0, T]
\end{array}
$$

where $\mathbf{b}$ are the body forces in the current configuration. Equation (2) expresses the mass conservation while equation (3) defines the momentum conservation. The Cauchy stress tensor can be decomposed into isotropic and deviatoric parts:

$\boldsymbol{\sigma}=-p \mathbf{I}+\boldsymbol{\tau}$

where $p=p(\mathbf{x}, t)$ is the pressure field, $\mathbf{I}$ is the identity tensor and $\tau$ is the deviatoric stress tensor. 
A suitable set of initial and boundary conditions must be provided to obtain a well-posed problem:

$\mathbf{u}(\mathbf{X}, t=0)=\mathbf{u}_{\mathbf{0}} \quad$ in $\Omega_{0}$

$\mathbf{u}(\mathbf{x}, t)=\tilde{\mathbf{u}}(\mathbf{x}, t) \quad$ on $\Gamma_{D} \times(0, T)$

$\boldsymbol{\sigma} \cdot \mathbf{n}=\mathbf{h}(\mathbf{x}, t) \quad$ on $\Gamma_{N} \times(0, T)$

$\rho(\mathbf{X}, t=0)=\rho_{0} \quad$ in $\Omega_{0}$

where $\mathbf{u}_{\mathbf{0}}, \tilde{\mathbf{u}}, \mathbf{h}, \rho_{0}$ are assigned functions, $\mathbf{n}$ is the outward normal to the boundary $\Gamma_{t}=\partial \Omega_{t}$, which is divided into two non-overlapping subsets $\Gamma_{D}$ and $\Gamma_{N}$, such that $\Gamma_{D} \cup \Gamma_{N}=\Gamma_{t}$ and $\Gamma_{D} \cap \Gamma_{N}=\emptyset$.

\subsection{Constitutive law}

Considering a Newtonian fluid, the deviatoric stress $\boldsymbol{\tau}$ and deviatoric strain rate $\boldsymbol{\epsilon}$ can be directly related through the fluid viscosity $\mu$ :

$\tau=2 \mu \epsilon$

where $\boldsymbol{\epsilon}$ is defined as:

$\boldsymbol{\epsilon}=\frac{1}{2}\left(\nabla_{\mathbf{x}} \mathbf{u}+\nabla_{\mathbf{x}} \mathbf{u}^{T}\right)-\frac{1}{3}\left(\nabla_{\mathbf{x}} \cdot \mathbf{u}\right) \mathbf{I}$

In many engineering applications (e.g. the simulation of landslides) the material is described as a non-Newtonian fluid according to Bingham law:

$\begin{array}{lrl}\boldsymbol{\tau}(\mathbf{u})=2 \mu \boldsymbol{\epsilon}(\mathbf{u})+\tau_{0} \frac{\boldsymbol{\epsilon}(\mathbf{u})}{\|\boldsymbol{\epsilon}(\mathbf{u})\|} & \text { if }\|\boldsymbol{\tau}\|>\tau_{0} \\ \boldsymbol{\epsilon}(\mathbf{u})=0 & \text { otherwise }\end{array}$

where $\|\cdot\|$ denotes the norms:

$\|\epsilon(\mathbf{u})\|=\sqrt{\frac{1}{2} \epsilon: \epsilon} \quad\|\boldsymbol{\tau}\|=\sqrt{\frac{1}{2} \boldsymbol{\tau}: \tau}$

The incrementally discontinuous behavior of equations (11) and (12), with infinite initial viscosity, introduces numerical difficulties which can be avoided using an exponential smoothing approximation $[35,9]$ :

$\boldsymbol{\tau}(\mathbf{u})=2 \tilde{\mu} \boldsymbol{\epsilon}(\mathbf{u})=\left[2 \mu+\frac{\tau_{0}}{\|\boldsymbol{\epsilon}\|}\left(1-e^{-n\|\boldsymbol{\epsilon}\|}\right)\right] \boldsymbol{\epsilon}(\mathbf{u})$

where the apparent viscosity $\tilde{\mu}$ has been introduced. When $n \rightarrow \infty$, the Bingham behaviour is recovered.

\subsection{Equation of state}

In the compressible framework, an equation of state which relates pressure $p$ and density $\rho$ is necessary to complete the problem definition. In particular, in the present work barotropic flows are considered and there is no dependence on temperature; consequently, as suggested in $[5,15]$, a modified Tait equation of state is used. In fact, although it was originally proposed for water, it has been proved that it is an appropriate equation to represent the behavior of a wide variety of other fluids and of some compressible solids [29]. The Tait equation of state expresses a direct relation between the pressure and density fields in the form:

$p(\rho)=p_{0}+K\left[\left(\frac{\rho}{\rho_{0}}\right)^{\gamma}-1\right]$

having defined the reference pressure $p_{0}$, reference density $\rho_{0}$, the specific heat ratio $\gamma$ and the bulk modulus $K$. Following [5], $\gamma=7$ is considered in all the examples of section 6 .

\section{Space discretization}

A standard Galerkin approach has been followed to discretize in space the equations (2)-(3). Let $S^{u}$ denote the space of admissible values for the velocity variables and $S^{\rho}$ the space of admissible values for density and pressure. Moreover let $S_{0}^{u}$ and $S_{0}^{\rho}$ be the corresponding test spaces compatible with homogeneous boundary conditions.

\subsection{Momentum conservation}

The weak form of the momentum equation (2) is obtained by using a vector test function $\mathbf{w} \in S_{0}^{u}$ and integrating over the domain $\Omega$ :

$$
\begin{array}{r}
\int_{\Omega_{t}} \mathbf{w} \cdot \rho \frac{d \mathbf{u}}{d t} d \Omega=\int_{\Omega_{t}} \mathbf{w} \cdot\left(\nabla_{\mathbf{x}} \cdot(\boldsymbol{\tau}-p \mathbf{I})+\rho \mathbf{b}\right) d \Omega \\
-\int_{\Gamma_{N}} \mathbf{w} \cdot((\boldsymbol{\tau}-p \mathbf{I}) \cdot \mathbf{n}-\mathbf{h}) d \Gamma \quad \forall \mathbf{w} \in S_{0}^{u}
\end{array}
$$

Then, applying the Green formula and using the decomposition of the stress tensor (4), the following expression can be obtained:

$$
\begin{aligned}
& \int_{\Omega_{t}} \mathbf{w} \cdot \rho \frac{d \mathbf{u}}{d t} d \Omega=\int_{\Omega_{t}} p\left(\nabla_{\mathbf{x}} \cdot \mathbf{w}\right) d \Omega \\
& -\int_{\Omega_{t}} 2 \bar{\mu} \nabla_{\mathbf{x}} \mathbf{w}: \boldsymbol{\epsilon} d \Omega \\
& +\int_{\Omega_{t}} \mathbf{w} \cdot \rho \mathbf{b} d \Omega+\int_{\Gamma_{N}} \mathbf{w} \cdot \mathbf{h} d \Gamma \quad \forall \mathbf{w} \in S_{0}^{u}
\end{aligned}
$$


where $\bar{\mu}=\mu$ for Newtonian fluids and $\bar{\mu}=\tilde{\mu}$ for nonNewtonian ones. Now introducing an isoparametric finite element discretization, velocity, pressure and density can be expanded in terms of nodal values as:

$$
\begin{aligned}
u_{i}(\mathbf{x}, t) & =\sum_{a} N_{a}^{u}(\mathbf{x}) U_{i a}(t) \\
p(\mathbf{x}, t) & =\sum_{a} N_{a}^{p}(\mathbf{x}) P_{a}(t) \\
\rho(\mathbf{x}, t) & =\sum_{a} N_{a}^{\rho}(\mathbf{x}) R_{a}(t)
\end{aligned}
$$

where $N^{u}, N^{p}$ and $N^{\rho}$ are the shape functions for velocity, pressure and density, $\mathbf{U}_{i}$ is the vector of nodal velocities in the i-th direction, $\mathbf{P}$ is the vector of nodal pressures and $\mathbf{R}$ contains the nodal values of the density. Substituting (18)-(20) in (16), the semi-discretized momentum equation is obtained:

$\mathbf{M}_{u} \frac{d \mathbf{U}}{d t}=-\mathbf{F}_{\text {int }}+\mathbf{F}_{\text {ext }}$

where the mass matrix $\mathbf{M}_{u}$ is defined as:

$\mathbf{M}_{u, a b}(t)=\int_{\Omega_{t}} \rho \mathbf{N}_{a}^{T}(\mathbf{x}) \mathbf{N}_{b}(\mathbf{x}) d \Omega$

with $\left(\mathbf{N}_{a}=\operatorname{diag}\left[N_{a}\right]\right)$ and the internal and external forces $\mathbf{F}_{\text {int }}$ and $\mathbf{F}_{\text {ext }}$ are respectively defined as:

$$
\begin{aligned}
F_{\text {int }, a i}(t)= & \int_{\Omega_{t}}(\boldsymbol{\tau}(\mathbf{x}, t)-p(\mathbf{x}, t) \mathbf{I})_{i j} \frac{\partial N_{a}^{u}(\mathbf{x})}{\partial x_{j}} d \Omega \\
F_{\text {ext }, a i}(t)= & \int_{\Omega_{t}} N_{a}^{u}(\mathbf{x}) \rho b_{i}(\mathbf{x}, t) d \Omega+ \\
& \int_{\Gamma_{N}} N_{a}^{u}(\mathbf{x}) h_{i}(\mathbf{x}, t) d \Gamma
\end{aligned}
$$

\subsection{Mass conservation}

Mass conservation (2) is enforced starting from the strong form $[36,13]$ :

$\rho(\mathbf{x}, t) J(\mathbf{x}, t)=\rho(\mathbf{X}, 0)=\rho_{0}(\mathbf{X})$

where $J(\mathbf{x}, t)=\operatorname{det} \mathbf{F}(\mathbf{x}, t)$ is the determinant of the deformation gradient $\mathbf{F}$. Multiplying equation (25) by a test function $q \in S_{0}^{\rho}$ and integrating over the domain $\Omega(t)$, the following expression is obtained:

$$
\begin{array}{r}
\int_{\Omega_{0}} \rho(\mathbf{x}, t) J(\mathbf{x}, t) q d \Omega_{0}=\int_{\Omega_{0}} \rho_{0}(\mathbf{X}) q d \Omega_{0} \\
\forall q \in S_{0}^{\rho}
\end{array}
$$

Introducing the finite element approximation (18)(20), the discretized problem reads:

$$
\mathbf{M}_{\rho}(t) \mathbf{R}=\mathbf{R}_{0}
$$

where $\mathbf{M}_{\rho}$ and $\mathbf{R}_{0}$ are defined as:

$$
\begin{aligned}
& \mathbf{M}_{\rho, a b}(t)=\int_{\Omega_{t}} N_{a}(\mathbf{x}) N_{b}(\mathbf{x}) d \Omega \\
& \mathbf{R}_{0, b}=\int_{\Omega_{0}} \rho_{0}(\mathbf{X}) N_{b}(\mathbf{X}) d \Omega_{0}
\end{aligned}
$$

From mass conservation equation (25), derives that, velocity mass matrix can be written in the reference configuration as:

$$
\begin{aligned}
\mathbf{M}_{u, a b}(\mathbf{x}, t)= & \int_{\Omega_{t}} \rho \mathbf{N}_{a}^{T}(\mathbf{x}) \mathbf{N}_{b}(\mathbf{x}) d \Omega= \\
& \int_{\Omega_{0}} \rho_{0} \mathbf{N}_{a}^{T}(\mathbf{X}) \mathbf{N}_{b}(\mathbf{X}) d \Omega_{0}
\end{aligned}
$$

Finally, the equation of state (15) can be enforced pointwise for the pressure nodal degrees of freedom:

$P_{a}=P_{0 a}+K\left[\left(\frac{R_{a}}{R_{0 a}}\right)^{\gamma}-1\right]$

where $P_{0}$ and $R_{0}$ are the reference nodal values of pressure and density, respectively.

\section{Time discretization}

Let us consider a generic subdivision of the time interval $[0, T]$ in $N$ time steps $\Delta t^{i}$ such that $T=\sum_{i=1}^{N} \Delta t^{i}$. Applying a first order Euler's forward scheme, the acceleration $\mathbf{a}^{n}$ at time $t^{n}$ can be written as:

$\mathbf{a}^{n}=\frac{\mathbf{u}^{n+1}-\mathbf{u}^{n}}{\Delta t^{n}}$

and the fully-discretized momentum conservation (21) at time $t^{n}$ reads:

$\mathbf{M}_{u}^{n+1} \mathbf{U}^{n+1}=\mathbf{M}_{u}^{n} \mathbf{U}^{n}+\Delta t^{n}\left(-\mathbf{F}_{i n t}^{n}+\mathbf{F}_{e x t}^{n}\right)$

Analogously, the mass conservation equation (25) becomes:

$\mathbf{M}_{\rho}^{n+1} \mathbf{R}^{n+1}=\mathbf{R}_{0}$

Finally, time integration of the equation of state (15) is straightforward:

$P_{a}^{n+1}=P_{0 a}+K\left[\left(\frac{R_{a}^{n+1}}{R_{0 a}}\right)^{\gamma}-1\right]$ 


\subsection{Explicit solution scheme}

From eq. (30) it can be noted that the mass matrix is defined on the reference configuration $\Omega_{0}$ and therefore it needs to be computed only when the reference configuration changes, as in the case of remeshing. If the same reference configuration $\Omega_{0}$ is used throughout the analysis, the mass matrix $\mathbf{M}_{u}$ needs to be computed only once at the beginning and then can be used for the rest of the analysis; if the reference configuration changes (for example due to a remeshing), the mass matrix should be recomputed. Consequently, within a time step the mass matrix is always constant $\mathbf{M}_{u}^{n+1}=\mathbf{M}_{u}^{n}$, so that the unknown velocity field $\mathbf{U}^{n+1}$, in (33) is expressed in terms of quantities referred to the previous time step and is given by:

$\mathbf{U}^{n+1}=\mathbf{U}^{n}+\left(\mathbf{M}_{u}^{n}\right)^{-1}\left(-\mathbf{F}_{i n t}^{n}+\mathbf{F}_{e x t}^{n}\right) \Delta t^{n}$

Moreover, matrix $\mathbf{M}_{u}$ can be lumped, leading to a diagonal system which can be solved node by node.

Similar considerations can be repeated also for the mass conservation equation. In fact, vector $\mathbf{R}_{0}$ is defined in the reference configuration (see eq. 29) and needs to be computed only when this configuration change Moreover, if also matrix $\mathbf{M}_{\rho}$ is lumped, a diagonal system is obtained also for equation (27).

An adaptive time step is used during the analysis. To respect the Courant Friedrichs Lewy (CFL) stability condition, a new stable $\Delta t$ is computed at every time step as:

$\Delta t=\beta \min _{e}\left(\frac{h_{e}}{c_{e}}\right)$

where $h_{e}$ is a characteristic element size computed runtime over the deformed element, $\beta$ a safety parameter and $c_{e}$ is the speed of sound defined as:

$c_{e}=\sqrt{\left.\frac{d p}{d \rho}\right|_{e}}=\sqrt{\frac{\gamma K}{\rho_{e}}}$

In $(38) \rho_{e}$ is the density on the element $e$ computed as the average of its nodal values. It must be noted that using a Tait's equation of state (15), density variations are usually limited (less than 1\%), practically leading to a constant value of the sound speed.

\section{PFEM solution scheme}

The system of fully discretized governing equations (33), (34), (35) is solved using a Lagrangian finite element approach based on the Particle Finite Element Method (PFEM). The PFEM has been extensively used to simulate not only free surface flows [21,34] and fluid-structure interaction problems $[22,10]$ but also problems dominated by interfaces between different phases or materials [20], tunneling [7], forming processes [31], melting of polymers [32], transport erosion and sedimentation in fluids [30]

The key features of the PFEM is the mesh adaptation. Adopting a Lagrangian scheme, nodes are moved following the fluid velocity and consequently the mesh soon becomes too distorted. Therefore, when a quality indicator shows that the mesh is deteriorated, a new mesh is generated using a Delaunay Triangulation based on the current node positions (only elements connectivities are modified). To respect internal and external boundaries and to identify the free surfaces, an alpha shape technique is adopted [14]. The solution scheme is sketched in Algorithm 1.

Linear shape functions are used for space discretization of both velocity and density. In fact, linear interpolation is based on nodal values of the unknowns only. Hence, since vertex nodes are not touched by the Delaunay triangulation, no mapping is necessary from the old mesh to the new one (see e.g. [21,10]). Higher order interpolations with additional nodes along the edges, which are not preserved by the triangulation, though possible in principle, would imply mapping the corresponding nodal quantities to new nodes.

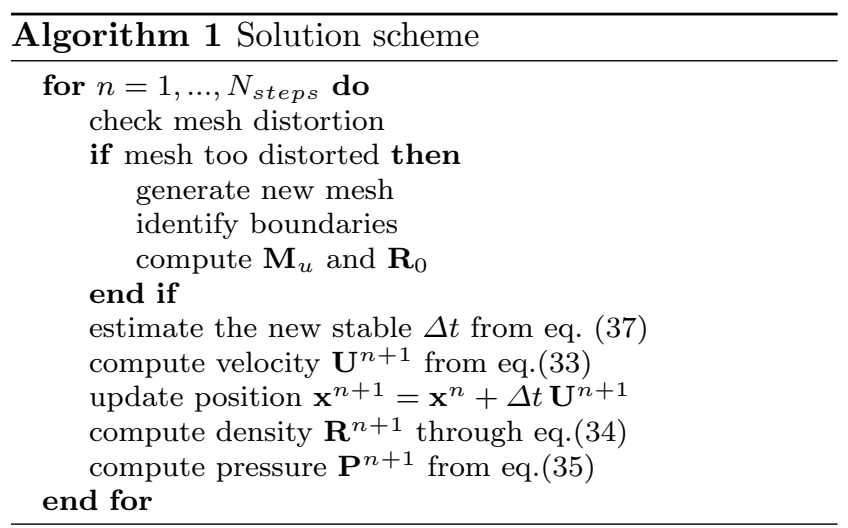

In standard implicit PFEM schemes, large part of the computing time is spent in the solution of the linear systems. On the contrary, in the proposed explicit approach no linear systems have to be solved, but obviously a smaller time step is necessary. As the computational burden for the solution of the linear systems but obviously, a smaller time step is necessary. As the computational burden for the solution of linear systems of the type involved in these analyses is usually proportional to $n(\log n), n$ being the number of degrees of freedom, it is expected that explicit schemes in large scale problems of short duration, where the computational 
cost increases approximately linearly with $n$, become more efficient. Moreover, the explicit scheme is particularly suited for parallelization. These aspects will be investigated in future works with particular emphasis on $3 \mathrm{D}$ cases. In the following section, some $2 \mathrm{D}$ small scale problems are considered to validate the proposed approach. For these small problems, an implicit scheme is however expected to be computationally more efficient.

\section{Numerical examples}

6.1 Sloshing of water in a prismatic tank

In the first test, the sloshing of a Newtonian fluid is presented. The geometry and the initial prescribed free surface proposed in [33] are considered (see Figure 1). The

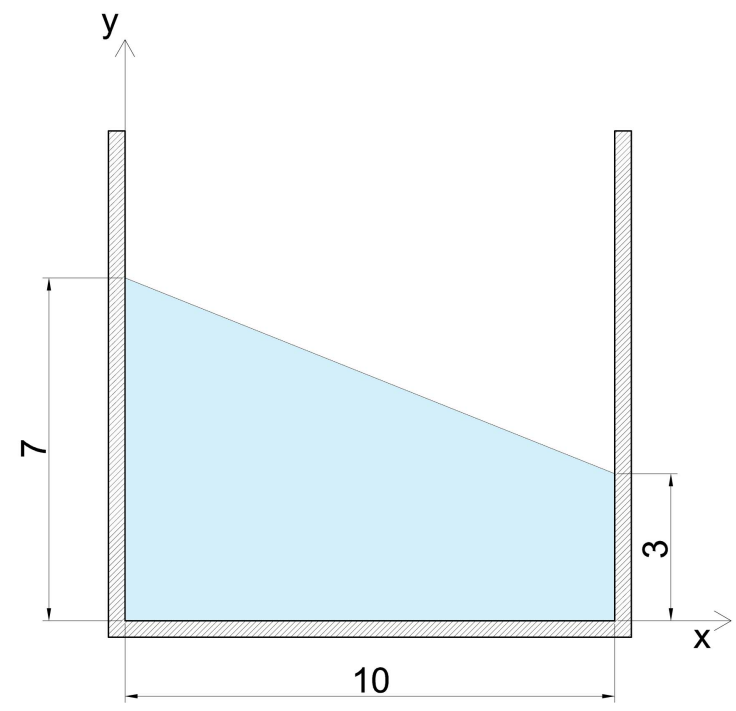

Fig. 1: Sloshing of water. Geometry of the problem. Dimensions in meters.

tank is filled with water whose constitutive parameters are: density $\rho_{0}=1000 \mathrm{~kg} / \mathrm{m}^{3}$, viscosity $\mu=10^{-3} \mathrm{~Pa} \mathrm{~s}$ and bulk modulus $K=2,210^{9} P a$. A mesh with 6008 nodes is considered. The stable time step size has to be recomputed whenever a re-triangulation is carried out and the used time step is therefore changing during the analysis. For this reason, only the average value of the time step will be reported for each example. In the present case, an average time step size of $2.16 \cdot 10^{-5} \mathrm{~s}$ has been used in the simulation. Figure 2 shows snapshots of the simulation at different time instants. The free surface profiles are practically coincident with the implicit incompressible formulation of [33], as can be appreciated in Figure 3.

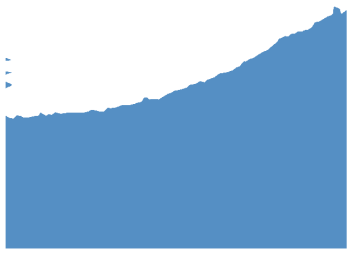

(a)

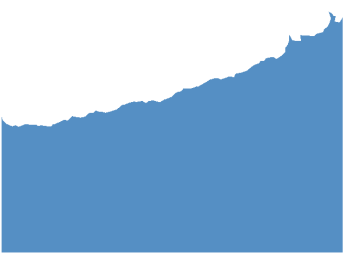

(c)

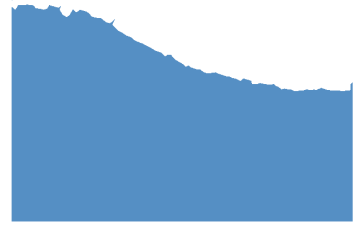

(b)

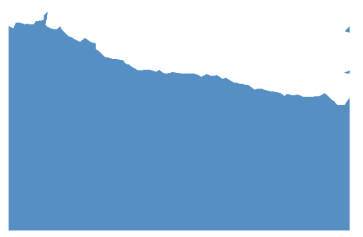

(d)
Fig. 2: Sloshing of water. Evolution of the free surface profile: (a) $t=5.7 \mathrm{~s}$; (b) $t=7.4 \mathrm{~s}$; (c) $t=13.3 \mathrm{~s}$; (d) $t=18.6 \mathrm{~s}$.

This test is used also to check the mass conservation properties of the proposed approach. It must be noted that in the standard PFEM for incompressible fluid, there are two different sources of mass variation: the inaccuracy of the solver in the solution of the mass conservation equation and the remeshing technique. In particular, numerical techniques used to stabilize equal order interpolation for pressure and velocity can increase the mass variation due to the unavoidable relaxation of the incompressibility constraint and the consequent error in the mass conservation equation. On the contrary, mass variations due to the remeshing are intrinsically connected to the PFEM. In general, they are unavoidable, but controllable by reducing the mesh size. A detailed description of these aspect can be found in [16]. The solution scheme proposed in this work does not produce any mass variation of the first type, since the density is computed at each step, so that the total mass is conserved exactly.

Figure 4 shows a comparison between numerical mass variation obtained with the present approach and obtained for the same problem with the optimized procedures proposed in [33]. While an excellent mass conservation is exhibited by the implicit approach in [33] (less than $2 \%$ ), zero mass variation is obtained with the 


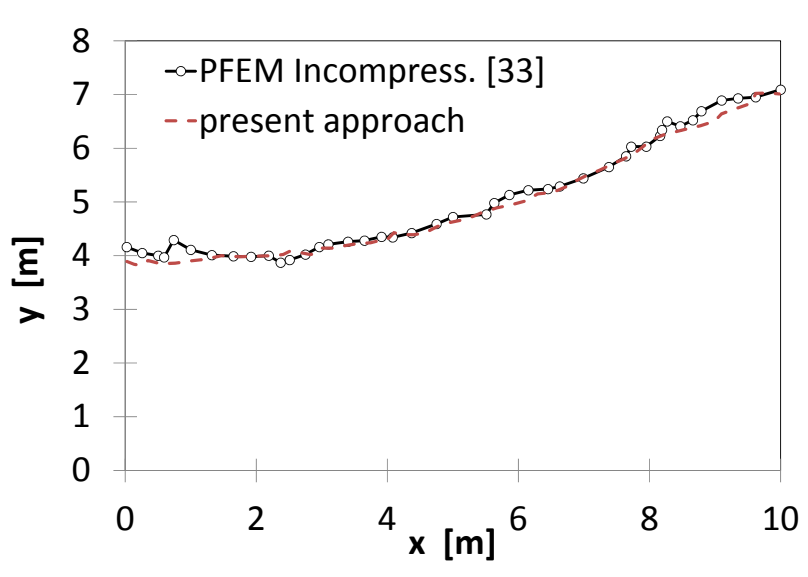

(a) $\mathrm{t}=5.7 \mathrm{~s}$

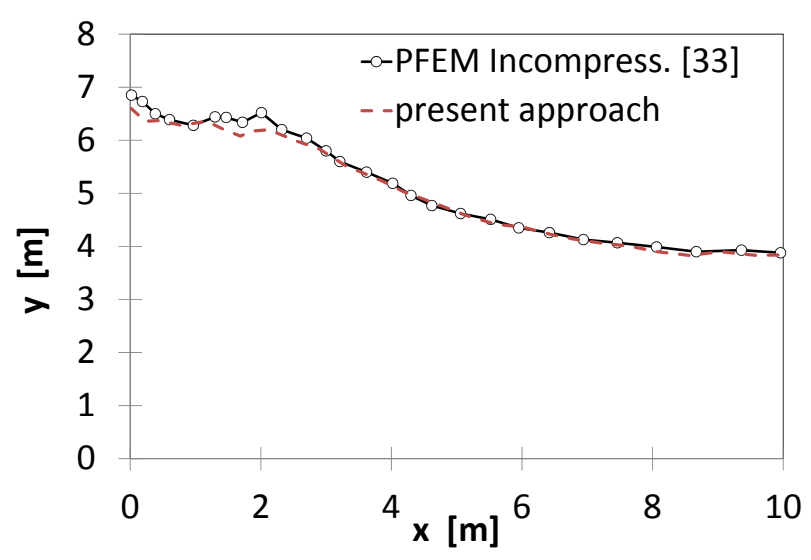

(b) $\mathrm{t}=7.4 \mathrm{~s}$

Fig. 3: Sloshing of water. Profile comparison at $t=5.7 \mathrm{~s}$ and $t=7.4 \mathrm{~s}$

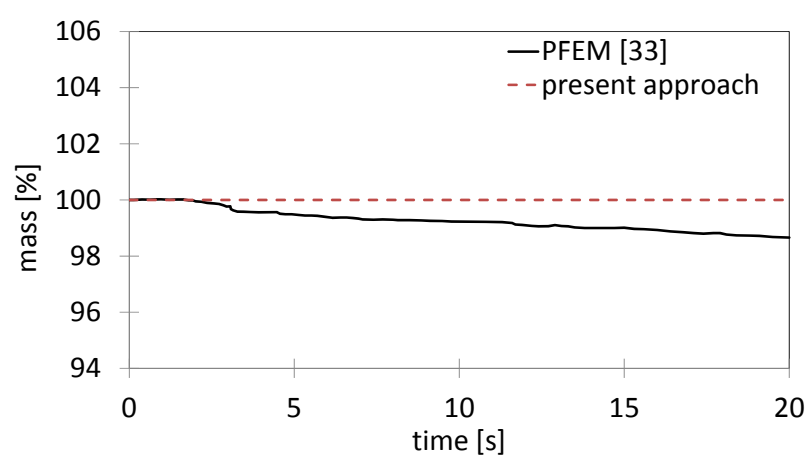

Fig. 4: Sloshing of water. mass variation due to the numerical approach; comparison with [33].

present approach. In should be noted that this latter result is independent of the time step size.

\subsection{Dam collapse on a rigid obstacle}

The simulation of the experimental dam break presented in [28] is here addressed. The experimental setting is depicted in figure 5: on the left side of the domain, a column of water is sustained by a rigid wall which simulates the presence of a dam. As the experiment starts, the wall is quickly removed, leaving the water able to flow in the tank under the effect of gravity. The flow impacts a rigid obstacle located in the middle of the box, with the formation of a long wave hitting the right wall. The constitutive parameters of the water used in the simulation are density $\rho_{0}=1000 \mathrm{~kg} / \mathrm{m}^{3}$, viscosity $\mu=10^{-3} \mathrm{~Pa}$ s and bulk modulus $K=2,210^{9} \mathrm{~Pa}$. The problem is solved with a mesh of 12862 nodes and an average time step size of $6.25 \cdot 10^{-7} \mathrm{~s}$.

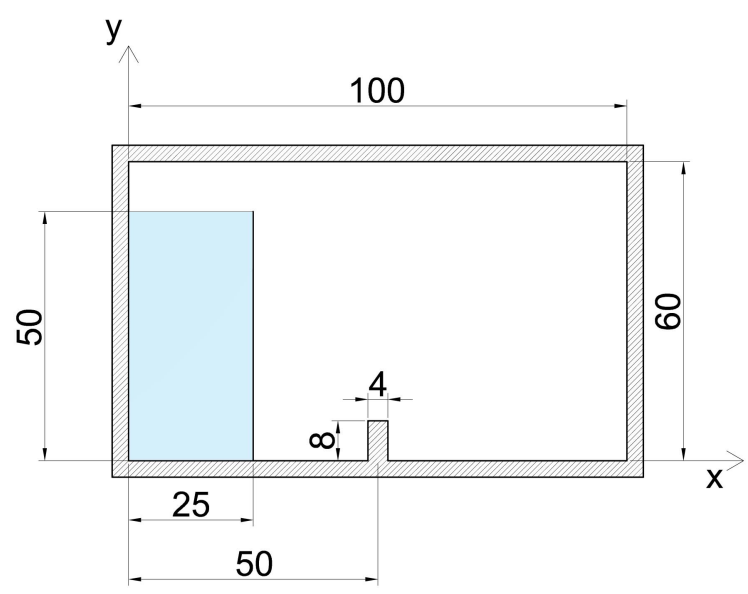

Fig. 5: Dam collapse on a rigid obstacle. Geometry of the problem. Dimensions in centimeters.

Figure 6 shows snapshots of the simulation at different time instants showing a good agreement both in terms of wave profile and timing with the experimental results of [28] and the simulations of [5].

In Figure 7 results are compared with the experimental test of [28] and the numerical results of [5] in terms of the free-surface profiles. In particular, [5] uses an ALE finite volume scheme in which the equations are solved in a Lagrangian form and then the solutions are mapped back on the original mesh. In the same graphs the results are also compared with the implicit incompressible formulation of the PFEM proposed in [23]. First of all, a higher level of the left part of the experimental water column can be noted in all the numerical results. That difference can be explained by the initial raise of the gate at the beginning of the prob- 


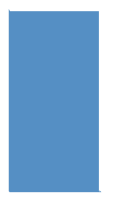

(a) $t=0 \mathrm{~s}$

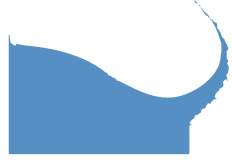

(c) $\mathrm{t}=0.26 \mathrm{~s}$

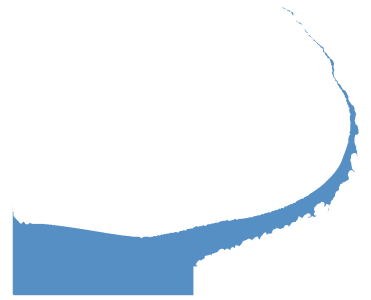

(e) $\mathrm{t}=0.40 \mathrm{~s}$ (b) $\mathrm{t}=0.13 \mathrm{~s}$
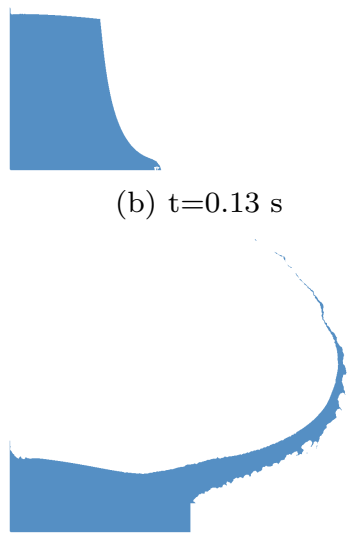

(d) $\mathrm{t}=0.39 \mathrm{~s}$

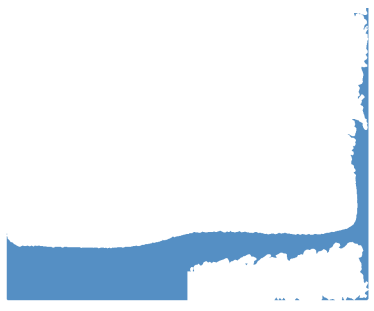

(f) $\mathrm{t}=0.50 \mathrm{~s}$

Fig. 6: Dam collapse on a rigid obstacle. Snapshots at different instants.

lem (as noted in [5]). This procedure is modeled as a boundary condition on the fluid velocity that is instantaneously released, while in the experiment it takes a short but finite interval of time, delaying the fall of the water column. Consequently, this difference affects the first stages of the simulation for all the numerical approaches, which indeed show a lower profile with respect to the experimental one.

Figure 7(a) shows that the present approach captures very accurately the real wave profile; in figure $7(\mathrm{~b})$ the numerical results show a slightly lower wave profile with respect to the real one but considering the high level of complexity of the phenomenon after the impact with the rigid obstacle, the comparison can be considered satisfactory. This may be due to the fact that in the physical test air is entrapped below the wave, whereas the air phase is not included in the present simulation.

\subsection{Fall of a water drop}

In this example, the free fall of a disk of water and its impact into a water basin at rest is considered. The geometry of the problem is sketched in Figure 8. Constitutive parameters are the same of the previous example,

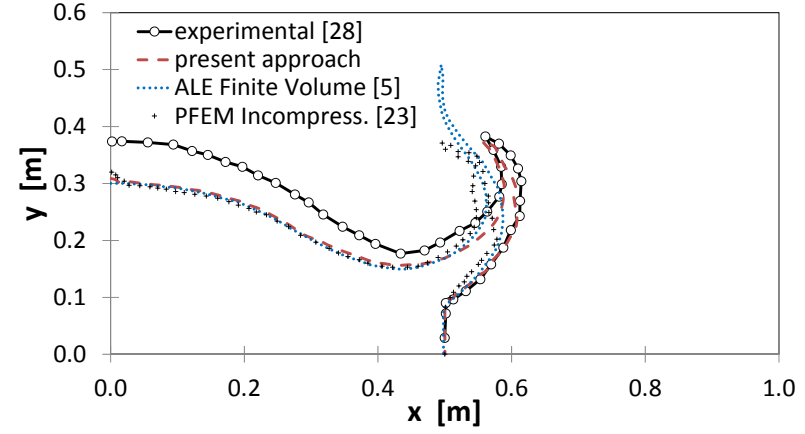

(a) $\mathrm{t}=0.26 \mathrm{~s}$

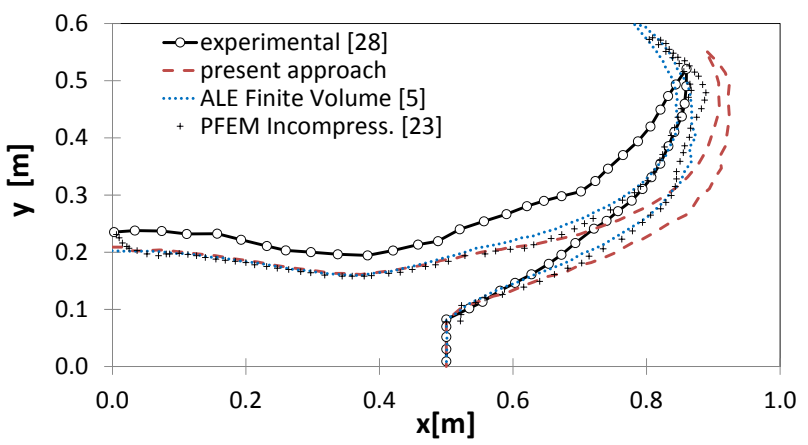

(b) $\mathrm{t}=0.39 \mathrm{~s}$

Fig. 7: Dam collapse with rigid obstacle. Profile comparison at $t=0.26 \mathrm{~s}$ and $t=0.39 \mathrm{~s}$

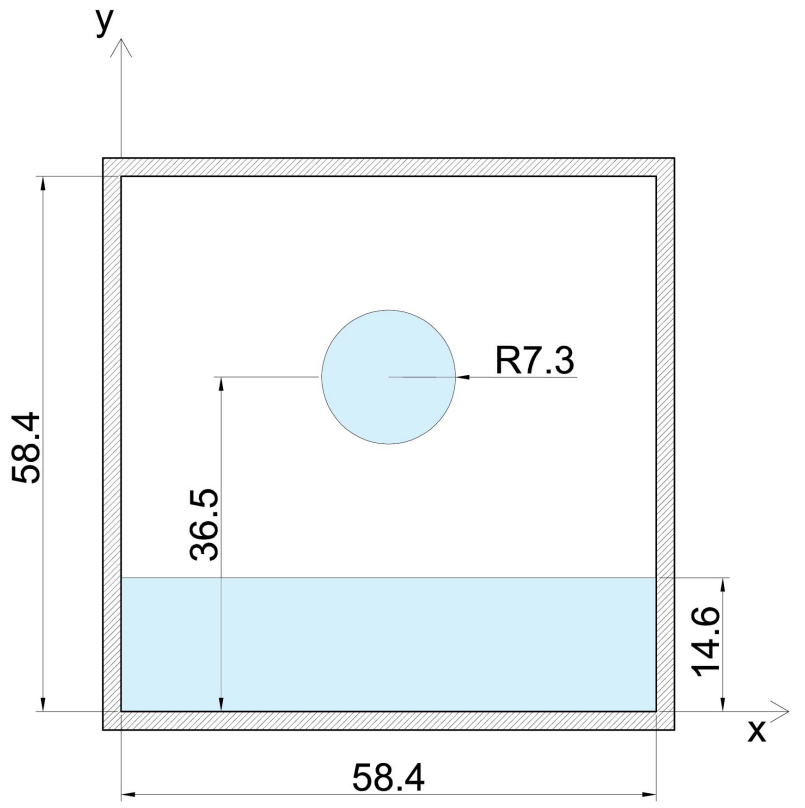

Fig. 8: Fall of a water drop. Geometry of the problem. Dimensions in centimeters.

a mesh of 13811 nodes has been used and the average time step size is $\Delta t=6.73 \cdot 10^{-7} \mathrm{~s}$. 
In Figure 9 the free surface profiles at synchronized instants are compared with the numerical results presented in [5]. Despite the completely different numerical approach, a very good agreement is observed.

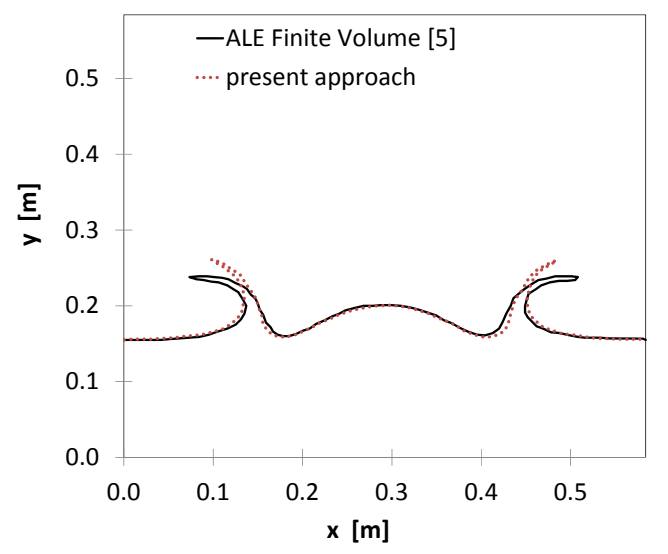

(a) $\mathrm{t}=0.229 \mathrm{~s}$

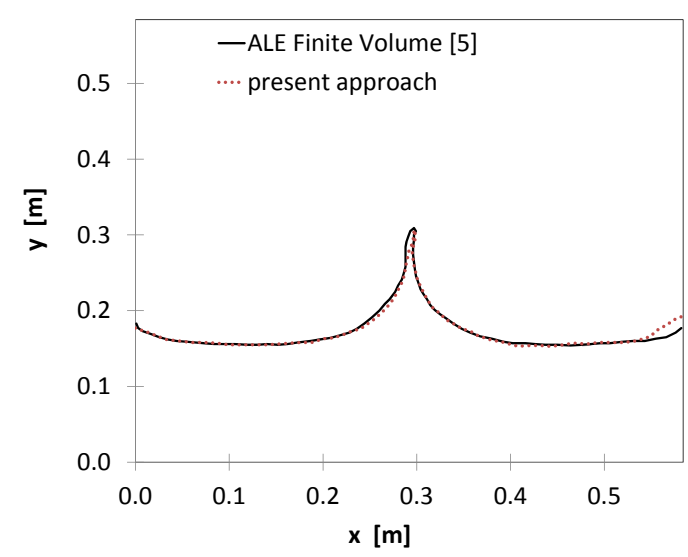

(b) $\mathrm{t}=0.648 \mathrm{~s}$

Fig. 9: Fall of a water drop. Free surface comparison at different time instants.

Figure 10 shows snapshots of the simulation at different time instants.

\subsection{Dam break of a Bingham fluid}

In this example, the dam break of a water-clay mixture presented in [27] is considered. Results obtained with the present numerical approach are compared with the experiment of [27] and the SPH results presented in [39]. The experimental setup is shown in Figure 11. The column of water-clay mixture has an initial length of $L=2 \mathrm{~m}$ and a height of $H=0,1 \mathrm{~m}$. It slides on a steep channel with a slope of $0,1 \%$.

The rheological properties used to model the material as a Bingham fluid are set as described in [39]:

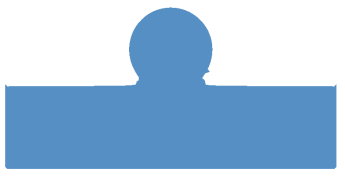

(a) $\mathrm{t}=0.177 \mathrm{~s}$

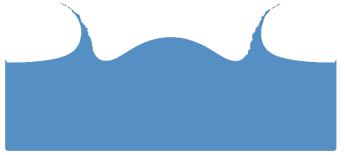

(c) $\mathrm{t}=0.229 \mathrm{~s}$

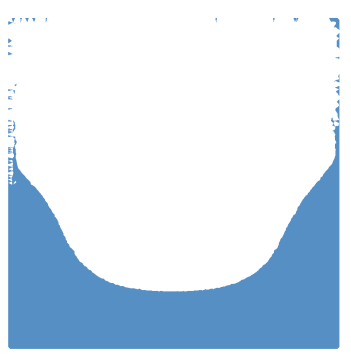

(e) $\mathrm{t}=0.349 \mathrm{~s}$

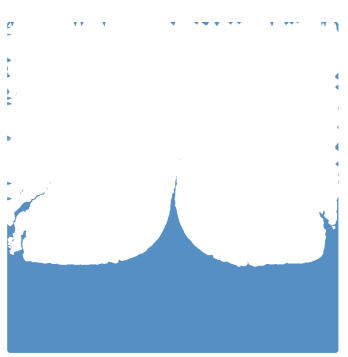

(g) $\mathrm{t}=0.618 \mathrm{~s}$

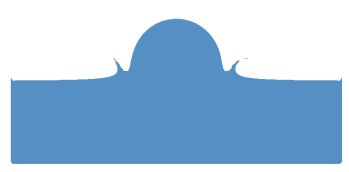

(b) $\mathrm{t}=0.194 \mathrm{~s}$

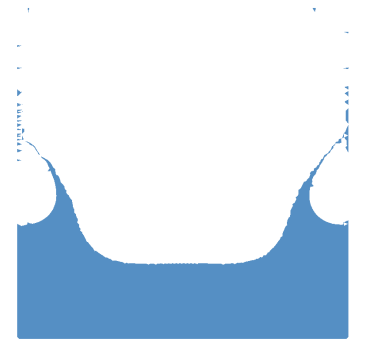

(d) $\mathrm{t}=0.290 \mathrm{~s}$

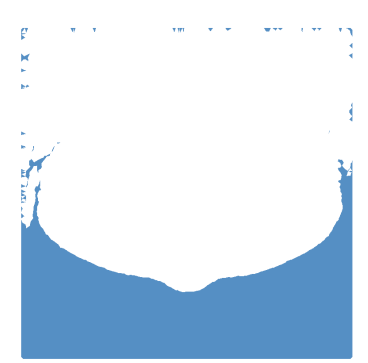

(f) $\mathrm{t}=0.545 \mathrm{~s}$

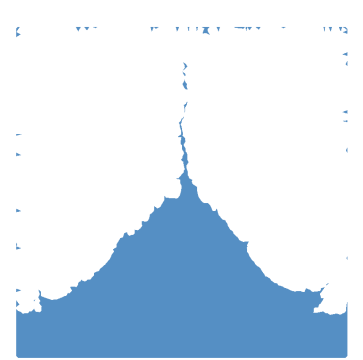

(h) $\mathrm{t}=0.859 \mathrm{~s}$
Fig. 10: Fall of a water drop. Snapshots at different time instants.

yield stress $\tau_{0}=25 P a$, viscosity $\mu=0.07 P a s$, density $\rho=1200 \mathrm{~kg}$ and bulk modulus is $K=2,510^{7} \mathrm{~Pa}$. The problem is solved with a mesh of 10261 nodes and an average time step size of $2.56 \cdot 10^{-6} \mathrm{~s}$. Snapshots at different time instants are shown in Figure 12.

The time evolution of the front distance obtained with the present approach is compared with the experimental test of [27] and the SPH results of [39]. The comparison shows a good agreement. The curve obtained with the present approach is slightly closer to the experimental one than the SPH curve of [39]. 


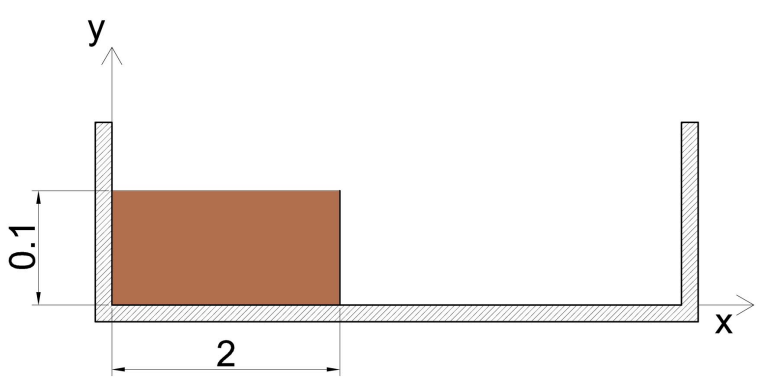

Fig. 11: Dam break of a Bingham fluid. Geometry of the problem. Dimensions in meters.

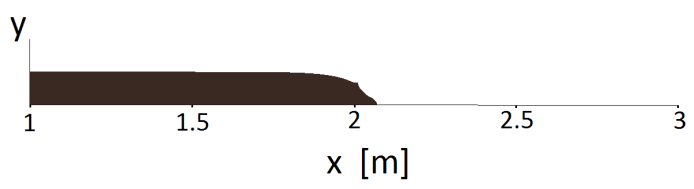

(a) $\mathrm{t}=0.10 \mathrm{~s}$

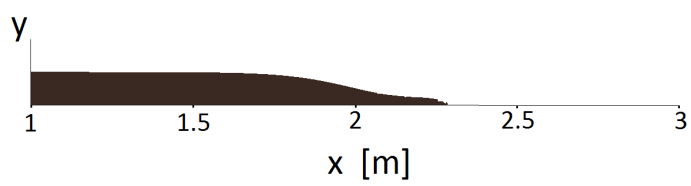

(b) $\mathrm{t}=0.30 \mathrm{~s}$

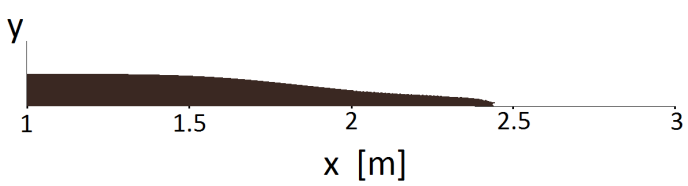

(c) $\mathrm{t}=0.60 \mathrm{~s}$

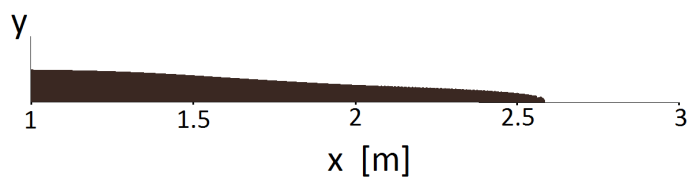

(d) $\mathrm{t}=1.00 \mathrm{~s}$

Fig. 12: Dam break of a Bingham fluid. Snapshots at different time instants.

Moreover, Figure 14 shows a comparison of the freesurface profiles between the present approach and the SPH results of [39].

\subsection{Debris flow on a water reservoir}

In this example, an experimental test performed at the Laboratory of Hydraulics, Hydrology and Glaciology (VAW) of the Swiss Institute of Technology (ETH) [18]

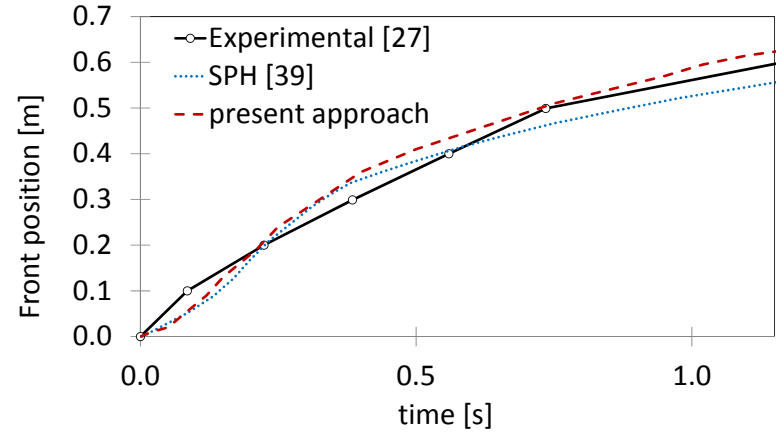

Fig. 13: Dam break of a Bingham fluid. Position of the mass front during time. Comparison between PFEM simulation, SPH simulation of [39] and experimental results [27]

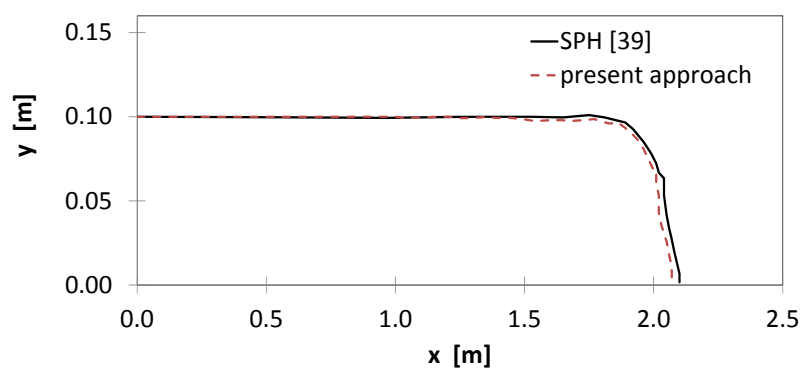

(a) $\mathrm{t}=0.10 \mathrm{~s}$

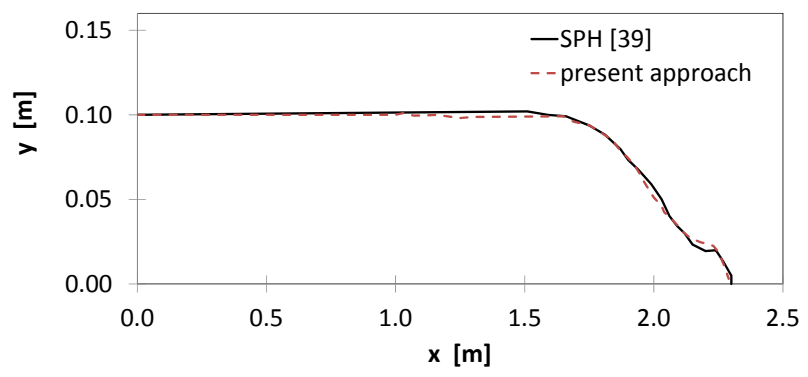

(b) $\mathrm{t}=0.60 \mathrm{~s}$

Fig. 14: Dam break of a Bingham fluid. Free surface comparison at different time instants.

is considered. In this test, a fast landslide impinges in a water reservoir. The reservoir consists of a rectangular prismatic water channel $11 \mathrm{~m}$ long, 0,5 $\mathrm{m}$ wide and $1 \mathrm{~m}$ high; a wave absorber at the end of the channel reduces wave reflections. The $3 \mathrm{~m}$ long ramp has a slide impact angle of $\theta=45^{\circ}$. At the top of the ramp a box filled with granular material is endowed with a pneumatic generator mechanism, which can accelerate the landslide box. The geometry is sketched in Figure 15.

As the experiment starts, the front flap of the box is opened and the granular material leaves the box with zero initial velocity, accelerates down the ramp due to gravity, and finally reaches the water basin generating 


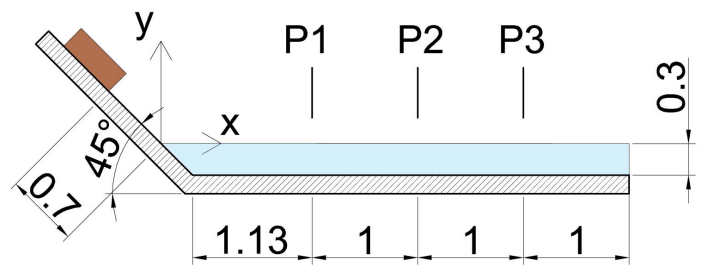

Fig. 15: Debris flow on a water reservoir.Geometry of the problem and position of the probes. Dimensions in meters.

an impulse wave in the channel. In the addressed experimental test, the entire volume of the box is filled with granular material, i.e. a rectangular prism whose dimensions are $0.6 \mathrm{~m}$ long, 0,236 $\mathrm{m}$ high and 0,472 $\mathrm{m}$ wide, with a total volume of landslide material of $0,0668 \mathrm{~m}^{3}$.

A Newtonian constitutive law is used to describe the water in the channel, whereas a non-Newtonian regularized Bingham fluid behavior is assumed for the sliding mass. All the materials properties are summarized in Table 1; an average time step size of $4.04 \cdot 10^{-6} \mathrm{~s}$ is used in the simulation.

\begin{tabular}{|c|c|}
\hline water density & $1000 \mathrm{~kg} / \mathrm{m}^{3}$ \\
\hline water viscosity & $0.001 \mathrm{~Pa} \mathrm{~s}$ \\
\hline average grain density & $\simeq 2650 \mathrm{~kg} / \mathrm{m}^{3}$ \\
\hline mixture density (soil and water) & $\simeq 1500 \mathrm{~kg} / \mathrm{m}^{3}$ \\
\hline viscosity of the debris flow & $75 \mathrm{~Pa} \mathrm{~s}$ \\
\hline yield stress of the debris flow & $35 \mathrm{~Pa}$ \\
\hline bulk modulus of the debris flow & $2.510^{7} \mathrm{~Pa}$ \\
\hline
\end{tabular}

Table 1: Debris flow on a water reservoir. Materials parameters.

The experimental and numerical results have been compared in terms of wave profiles. The data reported for the experimental test in [18] were recorded with seven Capacitance Wave Gages (CWG) disposed along the channel. The presence of the wave absorber in the physical model avoids phenomena of wave reflection, but it leads to numerical difficulties to simulate the fluid motion in the ending part of the channel. Therefore, it has been decided to focus on the wave profile recorded just by the first three probes. Figures 16(a),(b),(c) show the comparison between the evolutions of wave heights of the numerical and experimental results. The numerical results shown in [11] using an incompressible PFEM approach are also present in Figure 16. From the comparison, it can be noted that the results of the numerical approach are in good agreement in terms of the shape of the wave, of its maximum height and of the starting and stopping time.

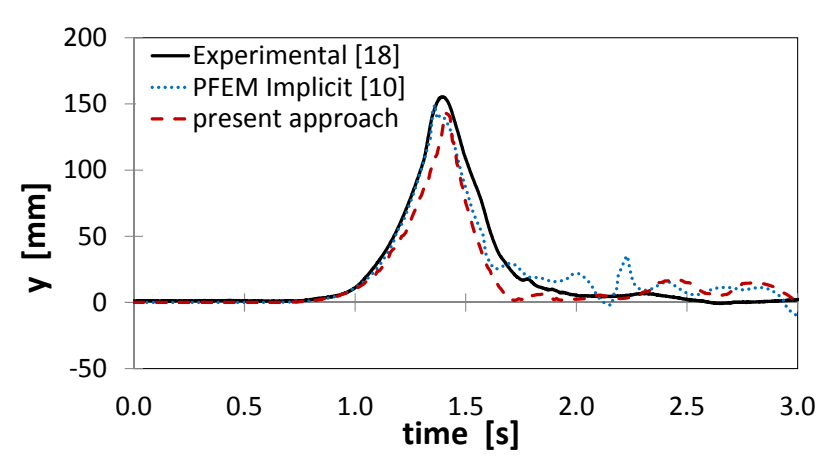

(a)

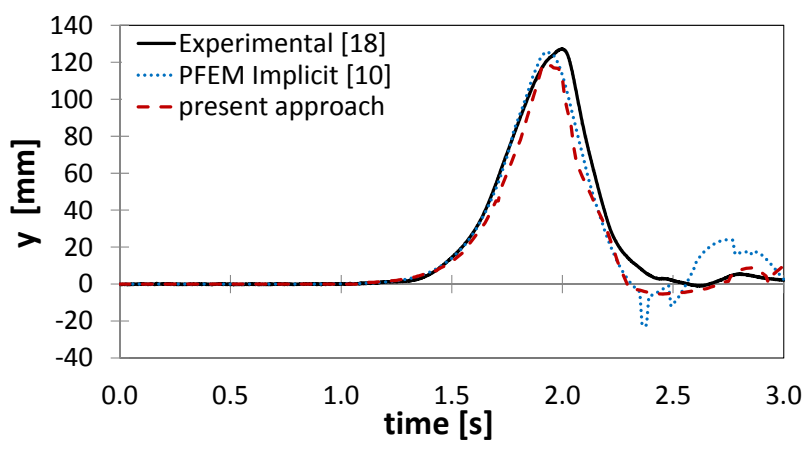

(b)

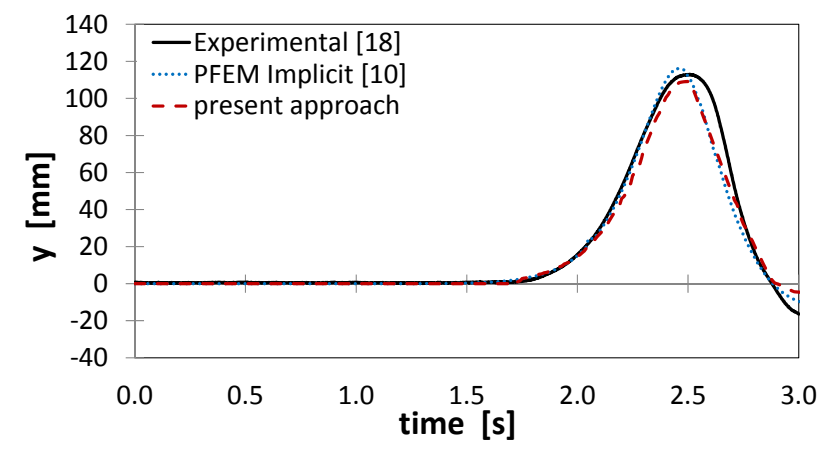

(c)

Fig. 16: Debris flow on a water reservoir. Comparisons of the wave height at the different probes.

Figure 17 shows snapshots of the simulation at different time instants.

\section{Conclusions}

This work proposes an explicit Langrangian finite element approach for the simulation of weakly compressible fluids in the presence of free surfaces. The compressible Navier-Stokes equations have been discretized with linear triangular finite elements using velocity and density as nodal unknowns. The Particle Finite Element Method has been used to update the configuration and to track the free surface evolution. 


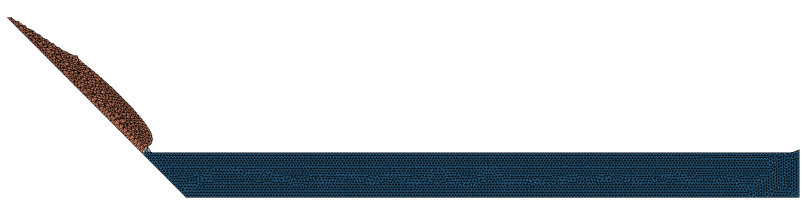

(a) $t=0.5 \mathrm{~s}$

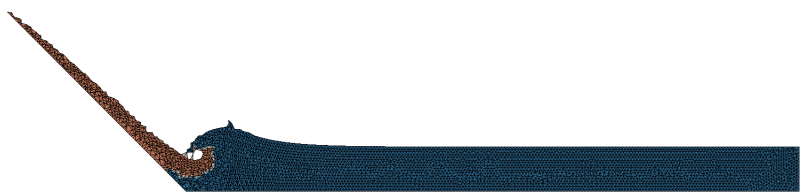

(b) $t=0.7 \mathrm{~s}$

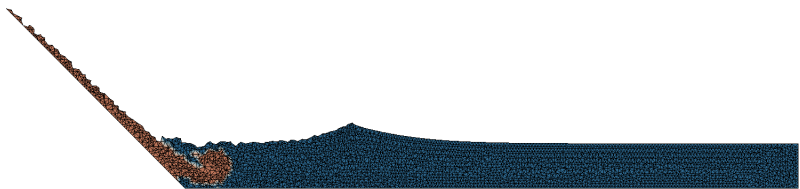

(c) $t=0.9 \mathrm{~s}$

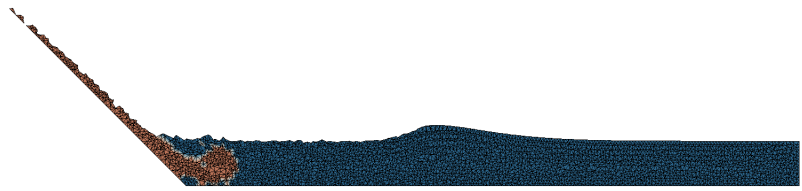

(d) $t=1.0 \mathrm{~s}$

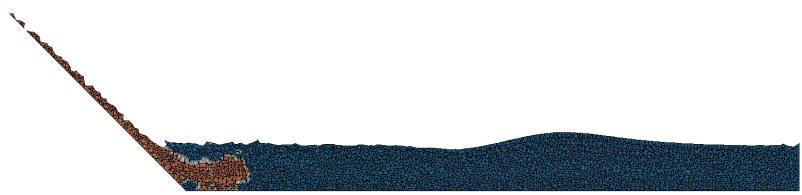

(e) $t=2.2 \mathrm{~s}$

Fig. 17: Debris flow on a water reservoir. Snapshots of the simulation at different time instants.

The proposed weakly compressible formulation does not produce any mass variation due to the numerical technique, since the density is computed at each step, so that the total mass is conserved exactly. Mass variations, decreasing with mesh refinement, are however expected due to the PFEM based boundary identification. An explicit time integration allows for a fast solution of the balance equations. Moreover, the explicit integration is particularly suited for a future parallelization of the code.

Different two dimensional numerical tests have been considered. The first examples are performed using water, while in the last two, a Bingham fluid is considered. Results have been compared with experimental data and with different numerical approaches, including implicit versions of the PFEM, showing a good agreement in all cases.
The considered two-dimensional tests imply only a limited computational cost. For this type of academic problems, an implicit approach is in general more competitive. The simulation of real scale engineering problems requires a general 3D framework that is currently under development. For large scale 3D problems, a significant computational gain is usually achieved by an explicit approach. This aspect, though, is to be verified in a forthcoming work.

Conflict of Interest The authors declare that they have no conflict of interest.

\section{References}

1. W. Bao and S. Jin. Weakly compressible high-order Istable central difference schemes for incompressible viscous flows. Computer Methods in Applied Mechanics and Engineering, 190(37-38):5009-5026, 2001.

2. A. J. Barlow. A compatible finite element multi-material ale hydrodynamics algorithm. International Journal for Numerical Methods in Fluids, 56(8):953-964, 2008.

3. D. J. Benson. Computational methods in lagrangian and eulerian hydrocodes. Computer Methods in Applied Mechanics and Engineering, 99(2-3):235 - 394, 1992.

4. A. Bernard-Champmartin, J.-P. Braeunig, and J.-M.l Ghidaglia. An eulerian finite volume solver for multimaterial fluid flows with cylindrical symmetry. Computers \& Fluids, 83:170 - 176, 2013. Numerical methods for highly compressible multi-material flow problems.

5. A. Bernard-Champmartin and F. De Vuyst. A low diffusive lagrange-remap scheme for the simulation of violent air-water free-surface flows. Journal of Computational Physics, 274:19-49, Oct 2014.

6. J.-P. Braeunig, B. Desjardins, and J.-M. Ghidaglia. A totally eulerian finite volume solver for multi-material fluid flows. European Journal of Mechanics - B/Fluids, 28(4):475 - 485, 2009.

7. J. M. Carbonell, E. Oñate, and B. Suarez. Modelling of tunnelling processes and cutting tool wear with the particle finite element method (pfem). Computational Mechanics, 52 (3):607-629, 2013.

8. Z. Chen, Z. Zong, M. B. Liu, and H. T. Li. A comparative study of truly incompressible and weakly compressible SPH methods for free surface incompressible flows. International Journal for Numerical Methods in Fluids, 73(9):813-829, jun 2013.

9. M. Cremonesi, L. Ferrara, A. Frangi, and U. Perego. Simulation of the flow of fresh cement suspensions by a lagrangian finite element approach. Journal of NonNewtonian Fluid Mechanics, 165:1555-1563, 2010.

10. M. Cremonesi, A. Frangi, and U. Perego. A lagrangian finite element approach for the analysis of fluid-structure interaction problems. International Journal of Numerical Methods in Engineering, 84:610-630, 2010.

11. M. Cremonesi, A. Frangi, and U. Perego. A lagrangian finite element approach for the simulation of waterwaves induced by landslides. Computer and Structures, 89:1086-1093, 2011. 
12. V.A. Dobrev, T.E. Ellis, T.V. Kolev, and R.N. Rieben. Curvilinear finite elements for lagrangian hydrodynamics. International Journal for Numerical Methods in Fluids, 65(11-12):1295-1310, 2011.

13. V.A. Dobrev, T.V. Kolev, and R.N. Rieben. High-order curvilinear finite element methods for lagrangian hydrodynamics. SIAM Journal on Scientific Computing, 34(5):B606-B641, 2012.

14. H. Edelsbrunner and E. P. Mucke. Three dimensional alpha shapes. ACM Transactions on Graphics, 13(1):4372, 1994.

15. C. Farhat, A. Rallu, and S. Shankaran. A higherorder generalized ghost fluid method for the poor for the three-dimensional two-phase flow computation of underwater implosions. Journal of Computational Physics, 227(16):7674-7700, 2008.

16. A. Franci and M. Cremonesi. Critical investigation of the particle finite element method. part i: volume conservation with remeshing. submitted for the pubblication, 2016.

17. S. Galera, P.-H. Maire, and J. Breil. A two-dimensional unstructured cell-centered multi-material $\{$ ALE $\}$ scheme using $\{\mathrm{VOF}\}$ interface reconstruction. Journal of Computational Physics, 229(16):5755 - 5787, 2010.

18. V. Heller. Landslide generated impulse waves: Prediction of near field characteristics. 2008.

19. T. J. R. Hughes, G. Scovazzi, and T. E. Tezduyar. Stabilized methods for compressible flows. Journal of Scientific Computing, 43(3):343-368, 2008.

20. S. R. Idelsohn, M. Mier-Torrecilla, and E. Oñate. Multifluid flows with the particle finite element method. Computer methods in applied mechanics and engineering, 198:2750-2767, 2009.

21. S. R. Idelsohn, E. Oñate, and F. Del Pin. The particle finite element method: a powerful tool to solve incompressible flows with free-surfaces and breaking waves. International Journal for Numerical Methods in Engineering, 61:964-989, 2004.

22. S. R. Idelsohn, E. Oñate, F. Del Pin, and N. Calvo. Fluid-structure interaction using the particle finite element method. Computer methods in applied mechanics and engineering, 195:2100-2113, 2006.

23. S.R. Idelsohn, J. Marti, A. Limache, and E. Oñate. Unified lagrangian formulation for elastic solids and incompressible fluids: Application to fluid-structure interaction problems via the pfem. Computer Methods in Applied Mechanics and Engineering, 197(19-20):1762-1776, mar 2008.

24. K. Kamran, R. Rossi, E. Oñate, and S.R. Idelsohn. A compressible lagrangian framework for modeling the fluid-structure interaction in the underwater implosion of an aluminum cylinder. Mathematical Models and Methods in Applied Sciences, 23(02):339-367, 2013.

25. K. Kamran, R. Rossi, E. Oñate, and S.R. Idelsohn. A compressible lagrangian framework for the simulation of the underwater implosion of large air bubbles. Computer Methods in Applied Mechanics and Engineering, 255(0):210 - 225, 2013.

26. I. J. Keshtiban, F. Belblidia, and M. F. Webster. Numerical simulation of compressible viscoelastic liquids. Journal of Non-Newtonian Fluid Mechanics, 122(1-3):131$146,2004$.

27. D. Komatina and M. Jovanovic. Experimental study of steady and unsteady free surface flows with water-clay mixtures. Journal of Hydraulic Research, 35(5):579-590, Sep 1997.
28. S. Koshizuka, H. Tamako, and Y. Oka. A particle method for incompressible viscous flow with fluid fragmentation. Computational Fluid Mechanics Journal, 113:134-147, 1995.

29. J.R. Macdonald. Some simple isothermal equations of state. Rewiews of Modern Physics, 38(4):669-679, 1966.

30. E. Oñate, M. A. Celigueta, S. R. Idelsohn, F. Salazar, and B. Suarez. Possibilities of the particle finite element method for fluid-soil-structure interaction problems. Computation mechanics, 48:307-318, 2011.

31. E. Oñate, A. Franci, and J. M. Carbonell. A particle finite element method for analysis of industrial forming processes. Computational Mechanics, 54:85-107, 2014.

32. E. Oñate, R. Rossi, S. R. Idelsohn, and K. Butler. Melting and spread of polymers in fire with the particle finite element method. International Journal of Numerical Methods in Engineering, 81 (8):1046-1072, 2010.

33. E. Oñate, A. Franci, and J. M. Carbonell. Lagrangian formulation for finite element analysis of quasiincompressible fluids with reduced mass losses. International Journal for Numerical Methods in Fluids, 74(10):699-731, 2014.

34. E. Oñate, S. R. Idelsohn, F. Del Pin, and R. Aubry. The particle finite element method. an overview. International Journal for Computational Methods, 1:267-307, 2004.

35. T. C. Papanastasiou. Flows of materials with yield. Journal of Rheology, 31(5):385-404, 1987.

36. G. Scovazzi, M.A. Christon, T.J.R. Hughes, and J.N. Shadid. Stabilized shock hydrodynamics: I. a lagrangian method. Computer Methods in Applied Mechanics and Engineering, 196(4-6):923-966, 2007.

37. G. Scovazzi, E. Love, and M.J. Shashkov. Multi-scale lagrangian shock hydrodynamics on $\mathrm{q} 1 / \mathrm{p} 0$ finite elements: Theoretical framework and two-dimensional computations. Computer Methods in Applied Mechanics and Engineering, 197(9-12):1056 - 1079, 2008.

38. M S. Shadloo, A. Zainali, M. Yildiz, and A. Suleman. A robust weakly compressible sph method and its comparison with an incompressible sph. International Journal for Numerical Methods in Engineering, 89(8):939-956, 2012.

39. S. Shao and E. Y.M. Lo. Incompressible sph method for simulating newtonian and non-newtonian flows with a free surface. Advances in Water Resources, 26(7):787800, July 2003.

40. J. Waltz, N.R. Morgan, T.R. Canfield, M.R.J. Charest, L.D. Risinger, and J.G. Wohlbier. A three-dimensional finite element arbitrary Lagrangian-Eulerian method for shock hydrodynamics on unstructured grids. Computers Fluids, 92(0):172 - 187, 2014

41. X. Xu and X.-L. Deng. An improved weakly compressible SPH method for simulating free surface flows of viscous and viscoelastic fluids. Computer Physics Communications, 201:43 - 62, 2016 